\title{
Micro-explosions in composite droplets: experimental observations and modelling
}

\author{
Sergei S Sazhin ${ }^{1 *}$, Tali Bar-Kohany ${ }^{2,3}$, Zuhaib Nissar ${ }^{4}$, Dmitrii Antonov ${ }^{5}$, Pavel A. Strizhak ${ }^{5}$, \\ Oyuna D Rybdylova ${ }^{1}$ \\ ${ }^{1}$ Advanced Engineering Centre, University of Brighton, Brighton BN2 4GJ, UK \\ ${ }^{2}$ School of Mechanical Engineering, Tel Aviv University, Tel Aviv, Israel \\ ${ }^{3}$ Mechanical Engineering Department, nren, Israel \\ ${ }^{4}$ Center for Automotive Research and Electric Mobility, Universiti Teknologi PETRONAS, \\ 32610 Seri Iskandar, Perak, Malaysia \\ ${ }^{5}$ National Research Tomsk Polytechnic University 30, Lenin Avenue, Tomsk 634050, Russia \\ ${ }^{*}$ Corresponding author: S.Sazhin@brighton.ac.uk
}

\begin{abstract}
Some of the most recent experimental and modelling results concerning the puffing/microexplosion of water-fuel composite droplets are presented and discussed. The experimental results refer to images of droplets during puffing/micro-explosion, times to puffing/micro-explosion and measurements of the temperatures inside the droplets. The model for puffing and microexplosion assumes that a small spherical water sub-droplet is located in the centre of a fuel droplet. The heat conduction equation is solved analytically inside this droplet at each time step, using the Robin boundary condition at its surface and the continuity conditions at the fuel-water interface. This analytical solution and an appropriate approximation of the nucleation temperature are incorporated into a numerical code in which droplet evaporation and the variable thermophysical properties are accounted for. It is assumed that the puffing/microexplosion process starts when the temperature between water and fuel reaches the nucleation temperature of water. The model predictions are shown to be consistent with available experimental data referring to the time to puffing/micro-explosion.
\end{abstract}

\section{Keywords}

Composite water/fuel droplets, micro-explosions, droplet heating/evaporation, Robin boundary conditions, nucleation temperature.

\section{Introduction}

It is well known that micro-explosions in water/fuel composite droplets, leading to their rapid disintegration, can substantially improve internal combustion engine performance, as discussed in [1]. The main aim of this paper is to summarise the most important recent experimental and modelling results obtained by the authors. In the models to be described the focus will be on capturing the most important features of the phenomenon (e.g. time to puffing/micro-explosion) leading to the development of relatively simple models of complex processes.

The most important previously obtained experimental results are summarised in the next section. This will be followed by an analysis of the previously suggested models and corrections/improvements made to them. Then the effects of replacing the boiling temperature of water with the nucleation temperature, as the temperature at which puffing/micro-explosion is expected to be initiated, is discussed. This will be followed by a summary of the main results of the paper.

\section{Experimental observations}

In this section we focus on the experimental results described in [1,2] which have been used for validation of the models described later.

The experiments described in [1] focus on relatively small droplets with diameters less than $250 \mu \mathrm{m}$. These experiments were conducted in a vessel with air at temperature $700 \mathrm{~K}$ and at 
atmospheric pressure. The time to puffing/micro-explosion was recorded with the help of a highspeed camera fitted with a long-distance microscope. The images from the microscope were processed to measure the initial droplet diameters and the time to puffing/micro-explosion. The experiments were performed using a resolution of $256 \times 800$ pixels $^{2}, 12,000$ frames per second, and exposure time of $2 \mu \mathrm{s}$. The uncertainty for the time to puffing/micro-explosion related to the identification of the frames for droplet creation and start of puffing $/ \mathrm{micro}$-explosion. The uncertainty for the measurement of the droplet diameter, related to the detection of the droplet perimeter, is estimated to be $\pm 37 \mu \mathrm{m}$. Droplets with diameters smaller than $50 \mu \mathrm{m}$ were ignored due to the experimental uncertainty. The time to puffing/micro-explosion was shown to increase with increasing initial droplet diameter.

In contrast to [1], the experiments described in [2] focus on much larger droplets with diameters in the range 1-3 $\mathrm{mm}$. Micro-explosions were shown to be the dominant mechanism of disintegration of composite fuel/water droplets in air at temperatures $850-1050^{\circ} \mathrm{C}$. It was shown that for all droplet initial diameters the maximal number of child droplets was observed when their diameters were close to $0.2 \mathrm{~mm}$. The time to puffing/micro-explosion was shown to stay almost the same when droplet initial diameters increased.

\section{Modelling approaches}

The most advanced models of puffing/micro-explosion processes are based on the Direct Numerical Simulation of the processes inside composite droplets, involving tracking the interfaces between water and fuel, water and water vapour, water vapour and fuel, and fuel and air. An example of such a model is described in [3]. The main problem with the application of these models to the analysis of the experimental data is that they require the identification of the exact initial location of water inside the fuel droplets. It would be very difficult, sometimes impossible, to do this in the experiments described above. Engineers working with this phenomenon are interested mainly in such characteristics as the time to puffing/micro-explosion, but not in the details of what happens inside the exploding droplet.

A simple model of the process, specifically focused on a crude approximation of this time, is described in [1]. The model described in this paper is based on the assumption that the fuel shell is spherically symmetric. It focuses on the heating of water and the fuel shell and is based on an application of the analytical solution to the heat transfer equation for the temperature inside a fuel/water droplet as a function of the distance from its centre and time. The temperature at the surface of the fuel ( $n$-dodecane) droplet was assumed to be equal to the fuel boiling temperature or slightly below this temperature (Dirichlet boundary condition). Based on the above-mentioned analytical solution, the time instant when the temperature at the water/fuel interface reaches the water boiling temperature was found. This temperature indicates the start of the puffing process quickly leading to micro-explosion. The effects of evaporation were ignored. This model uses a single formula for the estimation of the temperature at the water/fuel interface at any instant of time. Application of this formula to consecutive time instants allows one to estimate the required time to puffing/micro-explosion. The model predicts an increase in the latter time with increasing droplet diameter for droplets with diameters less than $250 \mu \mathrm{m}$. The model described in [4] is based on the same assumption regarding the geometry of the composite water/fuel droplet as the model described in [1]. The evolution of temperature inside the droplet in space and time is described by the following transient heat transfer equation:

$$
\frac{\partial T}{\partial t}=\kappa\left(\frac{\partial^{2} T}{\partial R^{2}}+\frac{2}{R} \frac{\partial T}{\partial R}\right)+P(t, R)
$$

where

$$
\kappa=\left\{\begin{array}{lll}
\kappa_{w}=k_{w} /\left(c_{w} \rho_{w}\right) & \text { when } \quad R \leq R_{w} \\
\kappa_{f}=k_{f} /\left(c_{f} \rho_{f}\right) & \text { when } \quad R_{w}<R \leq R_{d},
\end{array}\right.
$$

$\kappa_{w(f)}$ is the water (liquid fuel) thermal diffusivity, $k_{w(f)}$ is the water (liquid fuel) thermal conductivity, $c_{w(f)}$ is the water (liquid fuel) specific heat capacity, $\rho_{w(f)}$ is the water (liquid fuel) density, 
$R$ is the distance from the centre of the droplet, $t$ is time. The source term $P(t, R)$ takes into account volumetric droplet heating (e.g. heating due to thermal radiation).

The model is based on the analytical solution to Equation (1) with the following initial and boundary conditions, including the Robin boundary condition at the droplet surface:

$$
\begin{aligned}
& \left.T\right|_{t=0}=\left\{\begin{array}{lll}
T_{w 0}(R) & \text { when } \quad R \leq R_{w} \\
T_{f 0}(R) & \text { when } R_{w}<R \leq R_{d},
\end{array}\right. \\
& \left.T\right|_{R=R_{w}^{-}}=\left.T\right|_{R=R_{w}^{+}},\left.\quad k_{w} \frac{\partial T}{\partial R}\right|_{R=R_{w}^{-}}=\left.k_{f} \frac{\partial T}{\partial R}\right|_{R=R_{w}^{+}}, \\
& h\left(T_{\text {eff }}-T\left(R_{d}\right)\right)=\left.k_{f} \frac{\partial T}{\partial R}\right|_{R=R_{d}-0},
\end{aligned}
$$

where $h$ is the convection heat transfer coefficient,

$$
T_{\text {eff }}=T_{g}+\frac{\rho_{l} L \dot{R}_{d}}{h} .
$$

The term $\dot{R}_{d}=d R_{d} / d t$ takes into account the effect of evaporation, $h=k_{g} \mathrm{Nu} /\left(2 R_{d}\right)$, where the Nusselt number for stationary evaporating droplet is:

$$
\mathrm{Nu}=2 \frac{\ln \left(1+B_{T}\right)}{B_{T}},
$$

$B_{T}$ is the Spalding heat transfer number ${ }^{\star}$. It was assumed that $T_{w 0}\left(R_{w}\right)=T_{f 0}\left(R_{w}\right)$.

As in the case of the model described in [1], the start of puffing/micro-explosion was identified as the time instant when the temperature at the water/fuel interface reached the boiling temperature of water.

Unfortunately there was a mistake in the analytical solution obtained in [4]. This mistake did not affect the key conclusions drawn in [4], but affected the numerical values in the plots. The correct expression for the distribution of temperature inside the composite droplets, using the same assumptions as in [4], should have been as follows [5]:

$$
T=\frac{1}{R_{d} \xi}\left[\sum_{n=1}^{\infty} \Theta_{n}(t) v_{n}(\xi)+\frac{\mu_{0} \xi}{1+h_{0}}\right],
$$

where

$$
\begin{gathered}
\Theta_{n}(t)=\left(q_{n}+f_{n} \mu_{0}\right) \exp \left(-\frac{\lambda_{n}^{2} t}{R_{d}^{2}}\right)+\frac{p_{n}}{\lambda_{n}^{2}}\left[1-\exp \left(-\frac{\lambda_{n}^{2} t}{R_{d}^{2}}\right)\right], \\
f_{n}=\frac{1}{\left\|v_{n}\right\|^{2}} \int_{0}^{1} f(\xi) v_{n}(\xi) b \mathrm{~d} \xi \\
=\frac{1}{\left\|v_{n}\right\|^{2}}\left\{\int_{0}^{\xi_{w}} \frac{-\xi}{1+h_{0}} \frac{\sin \left(\lambda_{n} a_{w} \xi\right)}{\sin \left(\lambda_{n} a_{w} \xi_{w}\right)} k_{w} a_{w}^{2} \mathrm{~d} \xi+\int_{\xi_{w}}^{1} \frac{-\xi}{1+h_{0}} \frac{\sin \left(\lambda_{n} a_{f} \xi+\beta_{n}\right)}{\sin \left(\lambda_{n} a_{f} \xi_{w}+\beta_{n}\right)} k_{f} a_{f}^{2} \mathrm{~d} \xi\right\} \\
=\frac{1}{\left\|v_{n}\right\|^{2}}\left\{\int_{0}^{\xi_{w}} R_{d} \xi T_{0}\left(R_{d} \xi\right) \frac{1}{\sin \left(\lambda_{n} a_{w} \xi\right)} \int_{0}^{1} F_{0}(\xi) v_{n}(\xi) b \mathrm{~d} \xi\right. \\
\left.\left.p_{n}=\frac{1}{\left\|v_{n}\right\|_{w}^{2}} \int_{0}^{1} R_{w}^{2} \mathrm{~d} \xi+\int_{\xi_{w}}^{1} R_{d} \xi T_{0}\left(R_{d} \xi\right) \frac{\sin \left(\lambda_{n} a_{f} \xi+\beta_{n}\right)}{\sin \left(\lambda_{n} a_{f} \xi_{w}+\beta_{n}\right)} k_{f} a_{f}^{2} \mathrm{~d} \xi\right\}\right) b \mathrm{~d} \xi
\end{gathered}
$$

${ }^{*}$ The effect of evaporation on Nu was ignored in [4] where it was assumed that $\mathrm{Nu}=2$ 


$$
\begin{gathered}
=\frac{1}{\left\|v_{n}\right\|^{2}}\left\{\int_{0}^{\xi_{w}} R_{d}^{3} \xi \tilde{P}(\xi) \frac{\sin \left(\lambda_{n} a_{w} \xi\right)}{\sin \left(\lambda_{n} a_{w} \xi_{w}\right)} k_{w} a_{w}^{2} \mathrm{~d} \xi+\int_{\xi_{w}}^{1} R_{d}^{3} \xi \tilde{P}(\xi) \frac{\sin \left(\lambda_{n} a_{f} \xi+\beta_{n}\right)}{\sin \left(\lambda_{n} a_{f} \xi_{w}+\beta_{n}\right)} k_{f} a_{f}^{2} \mathrm{~d} \xi\right\} \\
f(\xi) \equiv \frac{-\xi}{1+h_{0}}=\sum_{n=1}^{\infty} f_{n}(t) v_{n}(\xi), \\
F_{0}(\xi) \equiv R_{d} \xi T_{0}\left(R_{d} \xi\right)=\sum_{n=1}^{\infty} q_{n}(t) v_{n}(\xi), \quad R_{d}^{3} \xi \tilde{P}(\xi)=\sum_{n=1}^{\infty} p_{n}(t) v_{n}(\xi), \\
v_{n}(\xi)=\left\{\begin{array}{l}
\frac{\sin \left(\lambda_{n} a_{w} \xi\right)}{\sin \left(\lambda_{n} a_{w} \xi_{w}\right)} \\
\frac{\sin \left(\lambda_{n} a_{f} \xi+\beta_{n}\right)}{\sin \left(\lambda_{n} a_{f} \xi_{w}+\beta_{n}\right)}
\end{array} \quad \text { when } \quad 0 \leq \xi \leq \xi_{w} \quad \xi_{w}<\xi \leq 1,\right.
\end{gathered}
$$

$\beta_{n}$ is $\beta\left(\lambda=\lambda_{n}\right)$,

$$
\begin{gathered}
\left\|v_{n}\right\|^{2}=\int_{0}^{1} v_{n}^{2} b \mathrm{~d} \xi=\frac{\sqrt{c_{w} \rho_{w} k_{w}}}{\lambda_{n} \sin ^{2}\left(\lambda_{n} a_{w} \xi_{w}\right)}\left[\frac{a_{w} \lambda_{n} \xi_{w}}{2}-\frac{\sin \left(2 a_{w} \lambda_{n} \xi_{w}\right)}{4}\right] \\
+\frac{\sqrt{c_{f} \rho_{f} k_{f}}}{\lambda_{n} \sin ^{2}\left(\lambda_{n} a_{f} \xi_{w}+\beta_{n}\right)}\left\{\frac{a_{f} \lambda_{n}\left(1-\xi_{w}\right)}{2}-\frac{\sin \left(2 \lambda_{n} a_{f}+2 \beta_{n}\right)-\sin \left(2 \lambda_{n} a_{f} \xi_{w}+2 \beta_{n}\right)}{4}\right\} \\
\beta=\cot ^{-1}\left[\frac{k_{f}-k_{w}}{k_{f} a_{f} \xi_{w} \lambda}+\frac{k_{w} a_{w}}{k_{f} a_{f}} \cot \left(a_{w} \lambda \xi_{w}\right)\right]+i \pi-a_{f} \lambda \xi_{w},
\end{gathered}
$$

$i=0,1,2,3, \ldots .$. , but we restrict the analysis to the case when $i=0$ (the values of $v$ would be the same for other values of $i$ ), and a countable set of positive eigenvalues $\lambda_{n}$ is obtained from the boundary condition at $\xi=1$ :

$$
\lambda_{n} a_{f} \cos \left(\lambda_{n} a_{f}+\beta\right)+h_{0} \sin \left(\lambda_{n} a_{f}+\beta\right)=0 .
$$

The following parameters were introduced:

$$
\begin{gathered}
H=\frac{h}{k_{f}}-\frac{1}{R_{d}}, \quad \mu=\frac{R_{d}}{k_{f}}\left(h T_{g}+\rho_{l} L_{f} \dot{R}_{d}\right), \quad \xi=R / R_{d}, \quad \tilde{P}(\xi)=P\left(\xi R_{d}\right), \\
F(t, \xi)=u(t, R) \equiv T(R, t) R, \quad h_{0}=H R_{d}=\frac{h R_{d}}{k_{f}}-1, \quad \mu_{0}=\mu R_{d}=\frac{R_{d}^{2}}{k_{f}}\left(h T_{g}+\rho_{l} L_{f} \dot{R}_{d}\right) .
\end{gathered}
$$

The above model with the correct analytical solution was applied in [5] to the analysis of heating/ evaporation/ puffing/ micro-explosion of the same composite n-dodecane/water droplet as in [4]. The droplet initial temperature and ambient gas temperature were taken as $300 \mathrm{~K}$ and 700 $\mathrm{K}$, respectively. The droplet initial radius was taken as $5 \mu \mathrm{m}$, the ambient pressure was set to $101.325 \mathrm{kPa}$ and the volume fraction of water $V_{w}$ was taken as $15 \%$ (these are the same parameters as used for Fig. 3 of [4]). It was shown that as the droplet heats-up and evaporates, $\xi_{w}$ shifts and tends to merge with the droplet surface. The changes in this parameter are attributed to shrinking of droplets due to evaporation. The effect of swelling was not taken into account. The time to puffing/micro-explosion for this particular case was shown to be equal to $0.135 \mathrm{~ms}$. This can be compared with the time to puffing/micro-explosion predicted by the previous algorithm but with the corrected analytical solution $(0.138 \mathrm{~ms})$ and time reported in [4] $(0.21 \mathrm{~ms})$. 


\section{Boiling versus nucleation temperature}

As mentioned in the previous section, the models developed in [1, 4] are based on the assumption that puffing starts when the temperature at the water/fuel interface reaches the water equilibrium boiling temperature $T_{B}$. At the same time, it is well known that, in the transient case when temperature increases with time quickly enough, boiling takes place not at the boiling temperature, but at the nucleation temperature $T_{N}$ which is higher than $T_{B}$. The link between these temperatures has been investigated in many papers, including $[7,8]$.

The authors of [8] suggested the following correlation for high heating rates:

$$
T_{N}=T_{B}+0.37 \cdot \dot{T}^{10 / J a_{H N}} \quad 10^{5} \leq \dot{T} \leq 10^{9} \quad \mathrm{~K} / \mathrm{s},
$$

where $J a_{H N}=626$ for water. Using the results presented in [7, 8], the following approximate formula, valid for intermediate to high heating rates, was obtained [5]:

$$
T_{N}=T_{N c}+160 \times \tanh \left(\dot{T} / 10^{5}\right) \quad 10^{2} \leq \dot{T} \leq 10^{6} \mathrm{~K} / \mathrm{s}
$$

where $T_{N c}=385 \mathrm{~K}$ corresponds to the nucleation temperature pertaining to the lower limit of the correlation $\left(10^{2} \mathrm{~K} / \mathrm{s}\right)$. All temperatures are in $\mathrm{K}$, and $\dot{T} \equiv d T / d t$ is in $\mathrm{K} / \mathrm{s}$. Formulae (7) and (8) were both obtained at atmospheric pressure.

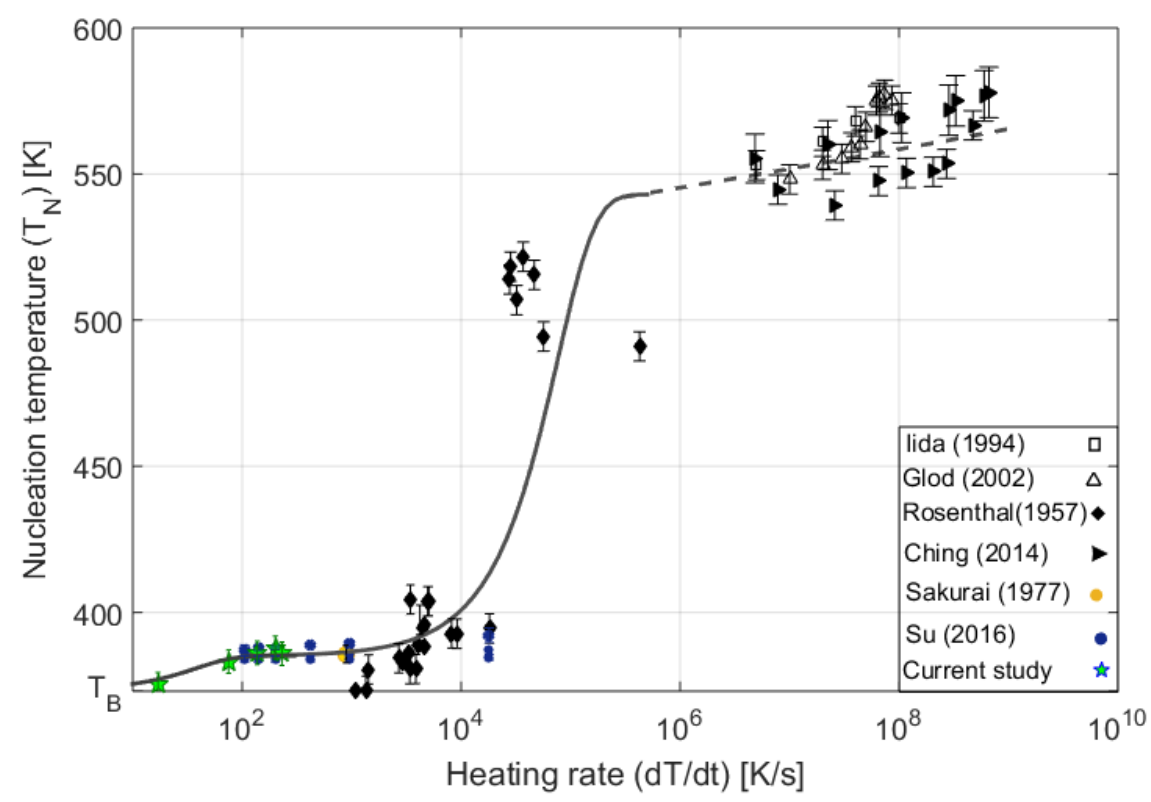

Figure 1. Nucleation temperature vs. heating rate - a comparison between experimentally observed values (see the insert) and those inferred from Equations (8) (solid curve) and (7) (dashed curve). The experimental points referred to as lida (1994), Glod (2002), Rosenthal (1957), Ching (2014), Sakurai (1977), and Su (2016) were obtained by various authors including [7,8] (see [5] for the references). The points referred to as Current study are taken from [5]. Reprinted from [5], Copyright Elsevier (2020).

Experimentally observed nucleation temperatures and those inferred from Equations (7) and (8) are shown in Figure 1. As follows from this figure, the agreement between the abovementioned correlations and experimental data can be considered to be reasonably good. Note that smaller droplets promote higher heating rates $(\dot{T})$ and higher nucleation temperatures $\left(T_{N}\right)$. For droplets with $R_{d 0}=5 \mu \mathrm{m}$ we obtain $\dot{T} \approx 10^{6} \mathrm{~K} / \mathrm{s}$ and the values of $T_{N}$ that were predicted by Eqs. (7) and (8) are more than $150 \mathrm{~K}$ above the boiling temperature of water.

In a series of experiments described in [5], the temperatures at which puffing/micro-explosion starts (identified as the nucleation temperature) and the values of $\dot{T}$ just before the start of puffing/micro-explosion were obtained. Kerosene (Jet A-1) was used as the fuel, and the compound water/fuel droplets at the initial temperatures $T_{d 0}=300 \pm 3 \mathrm{~K}$ were placed in a hot air. Three values of air temperature $T_{a}$ were used: $573 \mathrm{~K}, 673 \mathrm{~K}$ and $773 \mathrm{~K}$. Initial droplet radius 
$R_{d 0}=1.33 \pm 0.03 \mathrm{~mm}$, and water volume fraction $V_{w}=20 \%$ were used in the experiments which were performed at atmospheric pressure.

Two-colour LIP was used to determine the temperature at the water/fuel interface. This method is applicable for very slow heating rates and up to about $250-300 \mathrm{~K} / \mathrm{s}$. A special correlation was obtained for these low heating rates [5]:

$$
T_{N}=T_{B}+12 \times \tanh (\dot{T} / 50) ; \quad 0 \leq \dot{T} \leq 300 \mathrm{~K} / \mathrm{s}
$$

Good agreement between our experimental results, those reported in [7] and those predicted by (9) was demonstrated in [5]. The observed values of $\Delta T_{S H}$ (up to about $15 \mathrm{~K}$ ) need to be taken into account when modelling the puffing/micro-explosion phenomena.

The approach to determining time to puffing/micro-explosion suggested in [5] is based on determining the temperature $T_{w}$ and the rate of rise of this temperature $(\dot{T})$ at the water-fuel interface. Using the above-mentioned correlations, the latter parameter allowed the authors of [5] to find the time evolution of $T_{N}$ at the water-fuel interface. The intersection between the curves $T_{w}(t)$ and $T_{N}(t)$ gave the required time to puffing/micro-explosion $\left(\tau_{p}\right)$.

It was shown that the model tends to predict longer $\tau_{p}$ than observed in experiments. This might be related to the key assumption of our model that the water sub-droplet is located exactly in the centre of the fuel droplet. This assumption is questionable in the case of relatively large droplets, as considered in the experiments described in [5]. The difference in the time to puffing inferred from the assumptions that puffing starts at water boiling and nucleation temperatures needs to be taken into account, regardless of which model for puffing/micro-explosion is used in the analysis.

The model described in [5] was generalised in a non-self-consistent way by the authors of [9] to take into account the effect of gas velocity around droplets. In this generalisation the convection heat and mass transfer coefficients were inferred from the Abramzon and Sirignano model taking into account the non-zero velocity between droplets and gas [6]. At the same time the recirculation of the liquid inside the droplet was ignored.

In the model described in [10], the contribution of time required for bubble formation at the water-fuel interface to time to puffing/micro-explosion was taken into account alongside the contribution of the heating time of the composite droplet.

The results of the experimental and theoretical investigations of the mutual effect on their puffing/micro-explosion of droplets in a flow, using an example of two closely spaced droplets in tandem, are presented in [11].

\section{Conclusions}

Some of the most recent experimental and modelling results concerning the puffing/microexplosion of water-fuel droplets are discussed. The experimental results refer to images of droplets during puffing/micro-explosion, times to puffing/micro-explosion and measurements of the temperatures inside the droplets. Recently developed models for puffing/micro-explosion are based on the assumption that a small spherical water sub-droplet is located in the centre of a fuel droplet. In the latest model of the phenomenon, the heat conduction equation was solved inside this droplet at each time step, using the Robin boundary condition at its surface and the continuity conditions at the fuel-water interface.

The Abramzon and Sirignano model was used for the approximation of the droplet evaporation process and the effect of evaporation on the heating process was taken into account. It is pointed out that the above-mentioned model is based on the assumption that puffing/microexplosion starts at the time instant when the temperature at the water/fuel interface reaches the boiling temperature of water, while the start of this process should be associated with the time instant when the interface temperature reaches the water nucleation temperature $T_{N}$. The approximations for the nucleation temperature are reviewed. These approximations are shown to be consistent with experimental observations of $T_{N}$ (i.e. temperature at the water/fuel interface at the time instant when puffing/micro-explosion started) and $d T / d t$ (i.e. the rate of temperature increase just before the start of puffing/micro-explosion). 
The time to puffing/micro-explosion is predicted to be noticeably longer if puffing/micro-explosion takes place when the interface temperature is equal to the water nucleation temperature compared to the case when this temperature is equal to the boiling temperature of water. In both cases, for relatively large droplets $\left(R_{d 0} \approx 1.33 \mathrm{~mm}\right)$ the predicted times to puffing/microexplosion were larger that those observed experimentally. This is linked with the main limiting assumption of the model that the water sub-droplet is located exactly in the centre of the fuel droplet.

\section{Acknowledgements}

Work on this paper was supported by the Russian Science Foundation (Grant 21-19-00876) (contribution by S.S. Sazhin) the be UKRI Future Leaders Fellowship, UK (Grant no. MR/T043326/1) and the Royal Society (UK) (Grant no. IEC 192007) (contribution by O. Rybdylova), the Centre for Automotive Research and Electric Mobility (CAREM) of the Universiti Teknologi PETRONAS (UTP) (supported by the ministry of higher education Fundamental Research Grant Scheme (FRGS) (Grant FRGS/1/2017/TK10/UTP/01/2) and UTP Graduate Assistant (GA) studentship) (contribution by Z. Nissar), the National Research Tomsk Polytechnic University (project VIUISHFVP-184/2018) (contribution by P.A. Strizhak), and a Scholarship of the President of the Russian Federation (Grant SP-447.2021.1) (contribution by D.V. Antonov).

\section{Nomenclature}

$B_{T} \quad$ Spalding heat transfer number [-]

$c \quad$ specific heat capacity $[\mathrm{J} /(\mathrm{kg} \mathrm{K})]$

$h \quad$ convective heat transfer coefficient $\left[\mathrm{W} /\left(\mathrm{m}^{2} \mathrm{~K}\right)\right]$

$k \quad$ thermal conductivity $[\mathrm{W} /(\mathrm{m} \mathrm{K})]$

$L \quad$ latent heat of evaporation $[\mathrm{J} /(\mathrm{kg})]$

$\mathrm{Nu} \quad$ Nusselt number [-]

$P \quad$ radiation source term $[\mathrm{K} / \mathrm{s}]$

$R \quad$ distance from the droplet centre [m]

$t \quad$ time [s]

$T \quad$ temperature $[\mathrm{K}]$

$v_{n} \quad$ eigenfunctions [-]

$V_{w} \quad$ volume fraction of water [-]

\section{Greek symbols}

$\kappa \quad$ thermal diffusivity $\left[\mathrm{m}^{2} / \mathrm{s}\right]$

$\lambda_{n} \quad$ eigenvalues [-]

$\xi \quad R / R_{d}[-]$

$\rho \quad$ density $\left[\mathrm{kg} / \mathrm{m}^{3}\right]$

\section{Subscripts}

$B \quad$ boiling

$d \quad$ droplet

eff effective

$f \quad$ fuel (n-dodecane)

$g \quad$ ambient gas

$l \quad$ liquid

$N \quad$ nucleation

$s \quad$ surface

$w \quad$ water or water/fuel interface

$0 \quad$ initial conditions 


\section{References}

[1] Sazhin, S.S., Rybdylova, O., Crua, C., Heikal, M., Ismael, M.A., Nissar, Z., Aziz, A.R.B.A., 2019, International $J$ of Heat and Mass Transfer, 131, pp. 815-821.

[2] Antonov, D.V., Kuznetsov, G.V., Strizhak, P.A., Rybdylova, O., Sazhin, S.S., 2019, Microexplosion and autoignition of composite fuel/water droplets, Combustion and Flame, 210, pp. 479-489.

[3] Shinjo, J., Xia, J., Ganippa, L.C., Megaritis, A., 2014, , Phys. Fluids 26 (10), 103302,

[4] Nissar, Z., Rybdylova, O., Sazhin, S.S., Heikal, M., Aziz, A.R.B.A., Ismael, M.A., 2020, International J of Heat and Mass Transfer, 149, 119208.

[5] Sazhin, S.S., Bar-Kohany, T., Nissar, Z., Antonov, D., Strizhak, P., Rybdylova, O., 2020, International $J$ of Heat and Mass Transfer 161, 120238.

[6] Sazhin, S.S., 2014, "Droplets and Sprays", Springer, 2014.

[7] Su, G.-Y., Bucci, M., McKrell, T., Buongiorno, J., 2016, International J of Heat and Mass Transfer, 97, 667-684.

[8] Bar-Kohany, T., Amsalem, Y., 2018, International $J$ of Heat and Mass Transfer, 126, pp. 411-415.

[9] Antonov, D.V., Strizhak, P.A., Fedorenko, R.M., Nissar, Z., Sazhin, S.S., 2021, Fuel 289, 119814.

[10] Fostiropoulos, S., Strotos, G., Nikolopoulos, N., Gavaises, M., 2021, International J of Heat and Mass Transfer, 164, 120581.

[11] Antonov, D.V., Fedorenko, R.M., Strizhak, P.A., Castanet, G., Sazhin, S.S., 2021, International $J$ of Heat and Mass Transfer 176, 121449. 